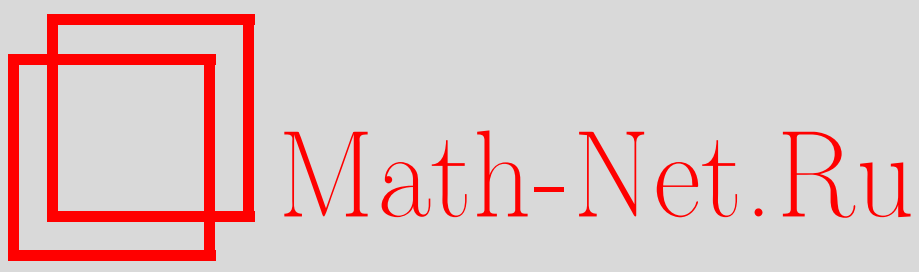

Д. В. Осипов, Бесконечномерный грассманиан Сато и когерентные пучки ранга 2 на кривых, Матем. сб., 2003, том 194, номер 11, 81-94

DOI: https://doi.org/10.4213/sm781

Использование Общероссийского математического портала Math-Net.Ru подразумевает, что вы прочитали и согласны с пользовательским соглашением

http://www . mathnet.ru/rus/agreement

Параметры загрузки:

IP: 3.85 .5 .30

26 апреля 2023 г., $11: 53: 12$ 
УДК 512.772.3

\title{
Д.В. Осипов \\ Бесконечномерный грассманиан Сато и когерентные пучки ранга 2 на кривых
}

\begin{abstract}
В работе исследуются образы (полу)стабильных пучков без кручения ранга 2 при отображении Кричевера в бесконечномерный грассманиан Сато.

Библиограффия: 8 названий.
\end{abstract}

Хорошо известно отображение Кричевера из множества пучков без кручения на кривых (с некоторыми дополнительными данными) в бесконечномерный грассманиан Сато (см., например, [1], [2]). Мы описываем при отображении Кричевера образы (полу)стабильных ранга 2 пучков без кручения на кривых и интерпретируем полученный результат в терминах действий некоторых однопараметрических подгрупп группы $\mathrm{SL}(2, k[[z]])$ на детерминантном расслоении бесконечномерного грассманова многообразия. Полученная формулировка аналогична конечномерному критерию Гильберта-Мамфорда из геометрической теории инвариантов (теорема 2).

Часть результатов этой статьи (без доказательств) была опубликована в заметке $[3]$.

Автор выражает благодарность М.С. Нарасиману за приглашение в международный центр теоретической физики в Триесте, где была получена часть результатов, а также глубокую благодарность А.Н. Паршину за постоянное внимание к работе и помощь.

\section{§1. Отображение Кричевера}

Рассмотрим следующие геометрические данные:

1) $C$ - приведенная неприводимая полная алгебраическая кривая, определенная над полем $k$;

2) $p$ - гладкая $k$-рациональная точка;

3) $\mathscr{F}$ - когерентный пучок без кручения $\mathscr{O}_{C}$-модулей ранга 2 на $C$;

4) $t_{p}$ - локальный параметр в точке $p$, т.е. $\widehat{\mathscr{O}}_{p}=k\left[\left[t_{p}\right]\right]$, где $\widehat{\mathscr{O}}_{p}-$ пополнение локального кольца $\mathscr{O}_{p}$ по максимальному идеалу;

5) $e_{p}$ - базис свободного модуля $\widehat{\mathscr{F}}_{p}$ ранга 2 над кольцом $\widehat{\mathscr{O}}_{p}$, где по определению $\widehat{\mathscr{F}}_{p} \stackrel{\text { def }}{=} \mathscr{F} \otimes_{\mathscr{O}_{C}} \widehat{\mathscr{O}}_{p}$.

Далее такой набор данных $\left(C, p, \mathscr{F}, t_{p}, e_{p}\right)$ мы будем называть көинтетом. Если эйлерова характеристика $\chi(\mathscr{F})=\mu$, то мы будем говорить, что квинтет имеет индекс $\mu$.

С другой стороны, рассмотрим $V=k((z)) \oplus k((z)), V_{0}=k[[z]] \oplus k[[z]]$. Тогда определим бесконечный грассманиан Cато $\mathrm{Gr}^{\mu}(V)$ как множество $k$-векторных

$$
\text { (С) Д. В. Осипов } 2003
$$


подпространств $W$ в $V$, являющихся фредгольмовыми индекса $\mu$, т.е. $\operatorname{dim}_{k} V /\left(V_{0}+\right.$ $W)<\infty, \operatorname{dim}_{k} W \cap V_{0}<\infty$ и $\operatorname{dim}_{k} W \cap V_{0}-\operatorname{dim}_{k} V /\left(V_{0}+W\right)=\mu$.

Определим отображение Кричевера $K$ из множества квинтетов индекса $\mu$ в $\mathrm{Gr}^{\mu}(V)$ как композицию следуюшей цепи очевидных отображений:

$$
\begin{gathered}
\left(C, p, \mathscr{F}, t_{p}, e_{p}\right) \rightarrow H^{0}(C \backslash p, \mathscr{F}) \hookrightarrow H^{0}\left(\operatorname{Spec} \mathscr{O}_{p} \backslash p, \mathscr{F}\right) \hookrightarrow H^{0}\left(\operatorname{Spec} \widehat{\mathscr{O}}_{p} \backslash p, \mathscr{F}\right) \\
=\widehat{\mathscr{F}}_{p} \otimes_{\widehat{\mathscr{O}}_{p}} \widehat{K}_{p} \stackrel{e_{p}}{\rightarrow}\left(\widehat{\mathscr{O}}_{p} \oplus \widehat{\mathscr{O}}_{p}\right) \otimes_{\widehat{\mathscr{O}}_{p}} \widehat{K}_{p} \stackrel{t_{p}}{\rightarrow} k((z)) \oplus k((z)),
\end{gathered}
$$

где $\widehat{K}_{p}$ - поле частных кольца $\widehat{\mathscr{O}}_{p}$.

Отображение Кричевера $K$ и его основные свойства подробно рассматривались многими авторами. Отметим здесь [1] в качестве алгебраического описания (но заметим, что определение в [1] несколько отличается от нашего определения) и [2] как аналитическое описание.

Для любого $W \in \mathrm{Gr}^{\mu}(V)$ определим кольцо

$$
A_{W} \stackrel{\text { def }}{=}\{f \in k((z)): f W \subset W\} .
$$

Лемма 1 (см. [1]). Для любого $W \in \mathrm{Gr}^{\mu}(V)$ и любого подкольиа $A \subset A_{W}$ такого, что $k \subset A$, мын имеем

1) $A \cap k[[z]]=k$;

2) если $A \neq k$, тогда кольцо $A$ имеет размерность 1 над $k$.

ДокАЗАтЕльство. Первое утверждение леммы следует из $\operatorname{dim}_{k} W \cap V_{0}<\infty$. Для доказательства второго утверждения рассмотрим следуюшую подгрупшу в группе целых чисел $\mathbb{Z}$ :

$$
\{n \in \mathbb{Z}: n=\nu(a), a \in \operatorname{Frac} A\}
$$

где $\nu$ - дискретное нормирование в поле $k((z))$ и $\operatorname{Frac} A$ - поле частных кольца $A$. Тогда эта подгруппа совпадает с $r \mathbb{Z}$ для некоторого целого $r$. Поэтому

1) сушествуют $f, g \in A$ такие, что $r=\nu(f)-\nu(g)$;

2) для любого целого $n$

$$
\operatorname{dim}_{k} A \cap z^{-n r} k[[z]] / A \cap z^{(-n+1) r} k[[z]] \leqslant 1
$$

Теперь из (2), утверждения 1) леммы и того, что $r$ является наибольшим общим делителем чисел $\nu(f)$ и $\nu(g)$, мы получаем

$$
\operatorname{dim}_{k} A / k[f, g]<\infty \text {. }
$$

Таким образом, размерность кольца $A$ над $k$ не больше двух. Если же предположить, что $f$ и $g$ - алгебраические элементы над $k$, то для всех пар целых чисел $n_{1}>0$ и $n_{2}>0$ элементы $f^{n_{1}} g^{n_{2}}$ включаются в один базис векторного пространства $A$ над $k$. Но последнее противоречит (2), и это противоречие завершает доказательство леммы.

Отметим следующее хорошо известное утверждение. 
Лемма 2. Элемент $W \in \mathrm{Gr}^{\mu}(V)$ находится в образе отображения Кричевера, если и только если $\operatorname{rank} A_{W}=1$, т.е. существуют два әлемента $f, g \in A_{W}$ такие, что числа $\nu(f)$ и $\nu(g)$ взаимно простьл.

ДокАЗАТЕльство. Отметим, что условие $\operatorname{rank} A_{W}=1$ эквивалентно следуюшему:

$$
\operatorname{dim}_{k} k((z)) /\left(A_{W}+k[[z]]\right)<\infty .
$$

Пусть $W=K\left(C, p, \mathscr{F}, t_{p}, e_{p}\right)$. Через $A_{C}$ обозначим образ кольца $H^{0}\left(C \backslash p, \mathscr{O}_{C}\right)$ в поле $k((z))$ :

$$
H^{0}\left(C \backslash p, \mathscr{O}_{C}\right) \hookrightarrow \widehat{K}_{p} \stackrel{t_{p}}{\rightarrow} k((z)) .
$$

Из теоремы Римана-Роха получаем

$$
\operatorname{dim}_{k} k((z)) /\left(A_{C}+k[[z]]\right)<\infty .
$$

И из $A_{C} \subset A_{W}$ следует (3).

Теперь пусть $\operatorname{rank} A_{W}=1$. Зафиксируем любое подкольцо $A \subset A_{W}$ так, что $k \subset A$ и $\operatorname{dim}_{k} A_{W} / A<\infty$. По лемме 1 кольцо $A$ имеет размерность 1 над $k$. Тогда из градуированной $k$-алгебры

$$
A_{*} \stackrel{\text { def }}{=} \bigoplus_{i=0}^{\infty} A \cap z^{-i} k[[z]]
$$

мы получаем полную неприводимую приведенную кривую $C=\operatorname{Proj} A_{*}$. И из (3) мы видим, что градуированный $A_{*}$-модуль

$$
W_{*} \stackrel{\text { def }}{=} \bigoplus_{i=-\infty}^{\infty} W \cap z^{-i} V_{0}
$$

определяет когерентный пучок без кручения $W_{*}^{\sim}$ ранга 2 на $C$. Более того, мы имеем $C=\operatorname{Spec} A \cup p$, где $p$-гладкая $k$-рациональная точка, $\widehat{\mathscr{O}}_{p}=k[[z]],\left.\mathscr{F}\right|_{C \backslash p}=$ $W^{\sim}, \widehat{\mathscr{F}}_{p}=V_{0}, t_{p}=z, e_{p}=(1,0),(0,1) \in V$. Кроме того,

$$
\begin{array}{ll}
\bigoplus_{i=0}^{\infty} H^{0}(C, \mathscr{O}(i p)) \simeq A_{*}, & \bigoplus_{i=-\infty}^{\infty} H^{0}(C, \mathscr{F}(i p)) \simeq W_{*}, \\
H^{0}(C, \mathscr{F}) \simeq W \cap V_{0}, & H^{1}(C, \mathscr{F}) \simeq V /\left(W+V_{0}\right) .
\end{array}
$$

Лемма доказана.

ЗАмЕчАниЕ. Если мы зафиксируем тройку данных $\left(C, p, t_{p}\right)$ и отождествим $\left(C, p, \mathscr{F}, t_{p}, e_{p}\right)$ с $\left(C, p, \mathscr{F}^{\prime}, t_{p}, e_{p}^{\prime}\right)$ для каждого изоморфизма пучков $\alpha: \mathscr{F} \rightarrow \mathscr{F}^{\prime}$ такого, что $\alpha\left(e_{p}\right)=e_{p}^{\prime}$, то отображение Кричевера - инъективное отображение. В этом случае $W \in \mathrm{Gr}^{\mu}(V)$ находится в образе такого отображения, если и только если $A_{C} W \subset W$.

Пусть теперь $W=K\left(C, p, \mathscr{F}, t_{p}, e_{p}\right) \in \mathrm{Gr}^{\mu}(V)$. Тогда с каждым подпучком $\mathscr{G}$ в $\mathscr{F}$ мы можем ассоциировать $k((z))$-векторное подпространство $L \mathscr{G} \subset V$ так, что $L_{\mathscr{G}} \cap W \neq 0:$

$$
\begin{gathered}
H^{0}(C \backslash p, \mathscr{G}) \hookrightarrow H^{0}(C \backslash p, \mathscr{F})=W \subset \mathrm{Gr}^{\mu}(V), \\
\mathscr{G} \longmapsto H^{0}(C \backslash p, \mathscr{G}) \times k((z)) \subset V .
\end{gathered}
$$

Приходим к следуюшему предложению. 
ПРЕДЛОЖЕНИЕ 1. Пусть $W=K\left(C, p, \mathscr{F}, t_{p}, e_{p}\right), R(\mathscr{F})-$ множество всех когерентных подпучков ранга $1 \mathscr{G} \subset \mathscr{F}$ таких, что пучок $\mathscr{F} / \mathscr{G}$ - когерентный пучок без кручения, $R(W)$ - множсество всех 1-мерных $k((z))$-векторных подпространств $L \subset V$ таких, что $L \cap W \neq 0$. Тогда отображсние (4) является взаимно однозначным соответствием межуд $R(\mathscr{F})$ u $R(W)$.

Более того, если $\mathscr{G} \in R(\mathscr{F}) \mapsto L_{\mathscr{G}} \in R(W)$, то

$$
\chi(C, \mathscr{G})=\operatorname{dim}_{k} W \cap L \mathscr{G} \cap V_{0}-\operatorname{dim}_{k} L \mathscr{G} /\left(W \cap L \mathscr{G}+L \mathscr{G} \cap V_{0}\right) .
$$

ДокАЗАТЕльство. Нетрудно видеть, что элементы множества $R(\mathscr{F})$ взаимно однозначно соответствуют 1-мерньм $k(\eta)$-векторным подпространствам в $\mathscr{F} \eta \stackrel{\text { def }}{=}$ $H^{0}(\operatorname{Spec} k(\eta), \mathscr{F})$, где $\eta \hookrightarrow C$ - обшая точка кривой $C$. Имеем каноническое вложение $\mathscr{F}_{\eta}$ в $V$, так как $H^{0}\left(\operatorname{Spec} \mathscr{O}_{p} \backslash p, \mathscr{F}\right)=\mathscr{F}_{\eta}-$ третий член в цепочке $(1)$. И это вложение задает нам взаимно однозначное соответствие между $R(\mathscr{F})$ и $R(W)$.

Более того, имеем следующую явную конструкцию. Пусть $L \in R(W)$. Тогда градуированньй $\left(A_{C}\right)_{*}$-модуль $(L \cap W)_{*} \stackrel{\text { def }}{=} \bigoplus_{i=-\infty}^{\infty}(L \cap W) \cap z^{-i} V_{0}$ определяет пучок без кручения ранга $1 \mathscr{G}_{L}=(L \cap W)_{*}^{\sim}$ на кривой $C=\operatorname{Proj}\left(A_{C}\right)_{*}$. Следовательно, отображение $L \rightarrow \mathscr{G}_{L}$ является обратньм к отображению $(4)$ и

$$
\begin{aligned}
& H^{0}\left(C, \mathscr{G}_{L}\right) \simeq W \cap L \cap V_{0}, \\
& H^{1}\left(C, \mathscr{G}_{L}\right) \simeq L /\left(W \cap L+L \cap V_{0}\right) .
\end{aligned}
$$

\section{§2. Детерминантное расслоение}

$\mathrm{Ha} \mathrm{Gr}^{\mu}(V)$ можно определить структуру алгебраической схемы, представляющей функтор, который обобщает обычный конечномерный грассманиан. (Это было явно сделано в [4].) Полученная схема будет приведенной и отделимой, и ее $k$-точки будут совпадать с множеством $\mathrm{Gr}^{\mu}(V)$, которое мы рассматривали в $\S 1$.

Опишем афинное открытое покрытие этой схемы. Пусть $A$ - соизмеримое с $V_{0}$ $k$-векторное подпространство $V$, т.е. $\operatorname{dim}_{k}\left(A+V_{0}\right) /\left(A \cap V_{0}\right)<\infty$. Определим

$$
\operatorname{Gr}_{A}^{\mu}(V) \stackrel{\text { def }}{=}\left\{W \in \mathrm{Gr}^{\mu}(V): V=A \oplus W\right\}
$$

Тогда $\operatorname{Gr}_{A}^{\mu}(V)$ можно отождествить с бесконечномерным афинным пространством, которое изоморфно спектру кольца многочленов от бесконечного множества переменных. И подсхемы $\operatorname{Gr}_{A}^{\mu}(V)$ определяют афинное открытое покрытие $\mathrm{Gr}^{\mu}(V)$, когда $A$ пробегает все соизмеримые с $V_{0}$ подпространства.

Можно определить линейное расслоение (детерминантное расслоение) Det на $\mathrm{Gr}^{\mu}(V)$ так, что для любого $W \in \mathrm{Gr}^{\mu}(V)$ геометрический слой имеет вид

$$
\operatorname{Det}_{W}=\bigwedge^{\max }\left(W \cap V_{0}\right) \otimes \bigwedge^{\max }\left(V / W+V_{0}\right)^{*} .
$$


Далее нам понадобится явное описание детерминантного расслоения Det, поэтому опишем его "в координатах". Для этого отождествим $k$-векторные пространства $V$ и $k((t))$ при помощи следующего непрерывного изоморфиизма:

$$
\begin{gathered}
V \longrightarrow k((t)) \\
\left(\sum a_{i} z^{i}\right) \oplus\left(\sum b_{i} z^{i}\right) \longmapsto \sum_{i}\left(a_{i} t^{2 i}+b_{i} t^{2 i+1}\right) .
\end{gathered}
$$

Отметим, что $V_{0}$ при этом изоморфизме переходит в $k[[t]]$.

Пусть $S_{\mu}$ - множество последовательностей $\left\{s_{-\mu+1}, s_{-\mu+2}, \ldots\right\}$ целых чисел таких, что

1) эти последовательности строго убывающие;

2) $s_{k}=-k$ для $k \gg 0$.

Отметим, что для каждого $\mu \in \mathbb{Z}$ множество $S_{\mu}$ находится во взаимно однозначном соответствии с множеством диаграмм Юнга.

Для каждого $S=\left\{s_{-\mu+1}, s_{-\mu+2}, \ldots\right\} \in S_{\mu}$ определим $H_{S} \in \operatorname{Gr}^{\mu}(V)$ как $k$-векторное пространство, порожденное элементами $\left\{t^{s_{k}}\right\}, A_{S} \subset k((t))$ - как наименьшее замкнутое $k$-векторное пространство, порожденное элементами $\left\{t^{q}: q \in\right.$ $\mathbb{Z} \backslash S\}$. Тогда

$$
\mathrm{Gr}^{\mu}(V)=\bigcup_{S \in S_{\mu}} \operatorname{Gr}_{A_{S}}^{\mu}(V) .
$$

Для любого $S \in S_{\mu}$ определим отображение $p_{S}: k((t)) \rightarrow H_{S}$ как проекцию, возникающую из разложения $H_{S} \oplus A_{S}=k((t))$. Через $0(\mu) \in S_{\mu}$ будем обозначать последовательность $\{\mu-1, \mu-2, \mu-3, \ldots\}$.

Приведем теперь описание детерминантного расслоения Det, взятое из [5; п. 7.7]. По данному $W \in \mathrm{Gr}^{\mu}(V)$ определим допустимый изоморфизм как изоморфизм

$$
w: H_{0(\mu)} \longrightarrow W, \quad w\left(t^{\mu-1}\right) \in W, \quad w\left(t^{\mu-2}\right) \in W, \quad \ldots
$$

с условием, что отображение

$$
p_{0(\mu)} \cdot w-\mathrm{Id}: H_{0(\mu)} \rightarrow H_{0(\mu)}
$$

имеет конечный ранг. (Заметим, что для любого $W \in \mathrm{Gr}^{\mu}(V)$ допустимые изоморфизмы всегда сушествуют.) Тогда детерминантное расслоение Det состоит из

$$
\{[\lambda, w]: \lambda \in k, w-\text { допустимый изоморфизм }\},
$$

отождествленных при помоши следуюшего отношения эквивалентности:

$$
[\lambda, w] \sim\left[\lambda \cdot \operatorname{det}\left(w^{\prime-1} \cdot w\right), w^{\prime}\right]
$$

где $w^{\prime}$ и $w$ - два допустимых изоморфизма для $W \in \mathrm{Gr}^{\mu}(V)$. (Отметим, что отображение

$$
w^{\prime-1} \cdot w-\operatorname{Id}: H_{0(\mu)} \rightarrow H_{0(\mu)}
$$


имеет конечный ранг как эндоморфизм $H_{0(\mu)}$, и поэтому $\operatorname{det}\left(w^{\prime-1} \cdot w\right)$ корректно определен по отношению к базисам $\left\{t^{\mu-1}, t^{\mu-2}, t^{\mu-3}, \ldots\right\}$.)

Пусть Det* - двойственное векторное расслоение к линейному расслоению Det. Для каждого $S \in S_{\mu}$ можно определить глобальное сечение

$$
\begin{gathered}
\pi_{S} \in H^{0}\left(\mathrm{Gr}^{\mu}(V), \operatorname{Det}^{*}\right): \text { Det } \longrightarrow k \\
{[\lambda, w] \stackrel{\pi_{S}}{\longrightarrow} \lambda \operatorname{det}\left(p_{S} \cdot w\right) \in k}
\end{gathered}
$$

при этом $p_{S} \cdot w-\mathrm{Id}: H_{0} \rightarrow H_{S}$ - отображение конечного $\operatorname{paнга.~Поэтому~} \operatorname{det}\left(p_{S} \cdot w\right)$ корректно определен по отношению к базисам

$$
\left\{t^{\mu-1}, t^{\mu-2}, \ldots\right\} \in H_{0(\mu)}, \quad\left\{t^{s_{-\mu+1}}, t^{s_{-\mu+2}}, \ldots\right\} \in H_{S}
$$

И как показано в [4] и [5], мы получаем замкнутое вложение (“плюккерово вложение"):

$$
\begin{gathered}
\operatorname{Gr}^{\mu}(V) \hookrightarrow \mathbb{P}\left(\Pi\left(S_{\mu}\right)^{*}\right) \stackrel{\text { def }}{=} \operatorname{Proj}\left(\operatorname{Sym}\left(\Pi\left(S_{\mu}\right)\right)\right), \\
W \longmapsto\left\{\pi_{S} \rightarrow \pi_{S}(w) \stackrel{\text { def }}{=} \operatorname{det}\left(p_{S} \cdot w\right)\right\}
\end{gathered}
$$

где $\Pi\left(S_{\mu}\right) \subset H^{0}\left(\mathrm{Gr}^{\mu}(V)\right.$, Det $\left.^{*}\right)$ - $k$-векторное подпространство, порожденное глобальньми сечениями $\left\{\pi_{S}: S \in S_{\mu}\right\}$, и каждая $k$-точка $W \in \operatorname{Gr}^{\mu}(V)$ определяет 1-мерное $k$-векторное подпространство $k \cdot\left\{\pi_{S} \mapsto \pi_{S}(w): S \in S_{\mu}\right\} \subset \Pi\left(S_{\mu}\right)^{*}$, не зависящее от выбора допустимого изоморфизма $w$ для $W$.

Отметим также, что $\pi_{S}(w) \neq 0$ тогда и только тогда, когда $W \in \mathrm{Gr}_{A_{S}}^{\mu}(V)$, т.е. $W \oplus A_{S}=V$.

Рассмотрим группу

$$
\mathrm{GL}\left(V, V_{0}\right) \stackrel{\text { def }}{=}\left\{g \in \operatorname{Aut}_{k}(V): \operatorname{dim}_{k}\left(g V_{0}+V_{0}\right) /\left(g V_{0} \cap V_{0}\right)<\infty\right\} .
$$

Отметим, что “в координатах" $\mathrm{GL}\left(V, V_{0}\right)$ соответствует групше обратимых элемен-

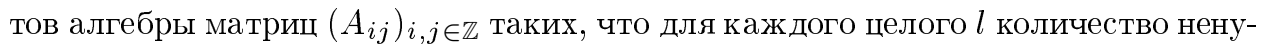
левых элементов $A_{i j}$ с $j \geqslant l, i \leqslant l$ - конечно. Действие $\left(A_{i j}\right)_{i, j \in \mathbb{Z}}$ на $k((t))$ следующее: $E_{i j}\left(t^{j}\right)=t^{i}$. (Здесь $E_{i j}$ - матрица с единицей на $(i, j)$-м месте и нулями во всех остальных местах.) Определим подгруппу

$$
\mathrm{GL}_{0}\left(V, V_{0}\right) \stackrel{\text { def }}{=}\left\{g \in \mathrm{GL}\left(V, V_{0}\right): \operatorname{dim}_{k} g V_{0} /\left(g V_{0} \cap V_{0}\right)=\operatorname{dim}_{k} V_{0} /\left(g V_{0} \cap V_{0}\right)\right\} .
$$

Тогда имеем очевидное действие $\mathrm{GL}_{0}\left(V, V_{0}\right)$ на $\mathrm{Gr}^{\mu}(V)$. Определим центральное расширение $\widehat{\mathrm{GL}_{0}}\left(V, V_{0}\right)$ группы $\mathrm{GL}_{0}\left(V, V_{0}\right)$ при помощи $k^{*}$ как группу всех автоморфизмов линейного расслоения Det на $\operatorname{Gr}^{0}(V)$, накрывающих действия элементов группы $\mathrm{GL}_{0}\left(V, V_{0}\right)$ на $\mathrm{Gr}^{0}(V)$. (Такое центральное расширение назьвается әруппой Мамфорда.) Приведем явное описание групшы $\widehat{\mathrm{GL}_{0}}\left(V, V_{0}\right)$ и ее действия на Det $\left.\right|_{\mathrm{Gr}^{\mu}(V)}$, взятое из [5]. Определим группу $H$ как следующую группу:

$$
\begin{aligned}
H=\left\{(g, E) \in \mathrm{GL}_{0}\left(V, V_{0}\right) \times \mathrm{GL}_{k}\left(t^{-1} k\left[t^{-1}\right]\right):\right. \\
\\
\left.g_{--}-E: t^{-1} k\left[t^{-1}\right] \rightarrow t^{-1} k\left[t^{-1}\right] \text { имеет конечный ранг }\right\},
\end{aligned}
$$


где $g_{--} \stackrel{\text { def }}{=}(g)_{i, j \leqslant-1}$. Пусть $N \subset H$ - нормальная подгруппа, определяемая как $N=\{(1, E) \in H: \operatorname{det} E=1\}$. Тогда $\widehat{\mathrm{GL}_{0}}\left(V, V_{0}\right)=H / N$ и проекция на первый сомножитель (см. определение $H$ ) задает отображение на $\mathrm{GL}_{0}\left(V, V_{0}\right)$. Теперь опишем действие $\widehat{\mathrm{GL}_{0}}\left(V, V_{0}\right)$ на Det $\left.\right|_{\mathrm{Gr}^{\mu}(V)}$.

Пусть $\mu=0$. Для $(g, E) \in H$ и $\left.[\lambda, w] \in \operatorname{Det}\right|_{\mathrm{Gr}^{0}(V)}$

$$
(g, E)[\lambda, w] \stackrel{\text { def }}{=}\left[\lambda, g w E^{-1}\right] .
$$

Последнее выражение определяет корректное действие $\widehat{\mathrm{GL}_{0}}\left(V, V_{0}\right)$ на Det $\left.\right|_{\mathrm{Gr}^{0}(V)}$.

Пусть $\mu \neq 0$. Определим действие $a \in \widehat{\mathrm{GL}_{0}}\left(V, V_{0}\right)$ на Det $\left.\right|_{\mathrm{Gr}^{\mu}(V)}$ как действие

$$
\sigma^{-\mu} \cdot \widetilde{\sigma}^{\mu}(a) \cdot \sigma^{\mu}
$$

где $\sigma:\left.\operatorname{Det}\right|_{\mathrm{Gr}^{\mu}(V)} \rightarrow$ Det $\left.\right|_{\mathrm{Gr}^{\mu-1}(V)}$ определяется по формуле $\sigma \cdot[\lambda, w]=\left[\lambda, t^{-1} \circ w\right]$; $t^{-1} \circ w: H_{0(\mu-1)} \rightarrow t^{-1} W, t^{-1} \circ w(x)=t^{-1} w(t x)$ допустимый, $x \in H_{0(\mu-1)}$; автоморфизм $\tilde{\sigma}: \widehat{\mathrm{GL}_{0}}\left(V, V_{0}\right) \rightarrow \widehat{\mathrm{GL}_{0}}\left(V, V_{0}\right)$ индуцирован следующим эндоморфизмом $H$ :

$$
(g, E) \longmapsto\left(t^{-1} g t, E_{\sigma}\right),
$$

где

$$
\left.E_{\sigma}\right|_{t^{-2} k\left[t^{-1}\right]}=t^{-1} E t, \quad E_{\sigma}\left(t^{-1}\right)=1 .
$$

Рассмотрим теперь группу $\Gamma=\mathrm{GL}(2, k[[z]])$, являющуюся подгруппой группы $\mathrm{GL}_{0}\left(V, V_{0}\right)$.

Лемма 3. Центральное расширение $\widehat{\mathrm{GL}_{0}}\left(V, V_{0}\right)$ расщепляется над Г.

ДокАЗАТЕЛЬСтво. Определим групповой гомоморфизм

$$
h: \Gamma \longrightarrow H, \quad h(\gamma)=\left(\gamma, \gamma_{--}\right), \quad \gamma \in \Gamma .
$$

(Заметим, что действие группы $\Gamma$ сохраняет $V_{0}$, и поэтому $\gamma_{--} \in \mathrm{GL}_{k}\left(k\left[t^{-1}\right]\right)$ ) Тогда гомоморфизм групा

$$
\Gamma \stackrel{h}{\longrightarrow} H \rightarrow H / N=\widehat{\mathrm{GL}_{0}}\left(V, V_{0}\right)
$$

расщепляет над Г центральное расширение.

В $V=k((z)) \oplus k((z))$ рассмотрим два подпространства (прямые):

$$
l_{1}=k((z)) \oplus 0, \quad l_{2}=0 \oplus k((z)),
$$

которые соответствуют $k\left(\left(t^{2}\right)\right)$ и $t k\left(\left(t^{2}\right)\right)$ в $k((t))$. Для любого $S \in S_{\mu}$ пусть

$$
n_{i}(S) \stackrel{\text { def }}{=} \operatorname{dim}_{k} H_{S} \cap V_{0} \cap l_{i}-\operatorname{dim}_{k} l_{i} /\left(H_{S} \cap l_{i}+l_{i} \cap V_{0}\right), \quad i=1,2 .
$$

Отметим, что

$$
n_{1}(S)+n_{2}(S)=\mu \text {. }
$$

Пусть $\left(\begin{array}{cc}\alpha^{a_{1}} & 0 \\ 0 & \nu^{a_{2}}\end{array}\right) \in \Gamma, \alpha, \nu \in k^{*}, a_{i} \in \mathbb{Z}$. По лемме 3 мы можем рассмотреть действие $\left(\begin{array}{cc}\alpha^{a_{1}} & 0 \\ 0 & \nu^{a_{2}}\end{array}\right)$ на детерминантном расслоении Det. 
ПРЕДЛОЖЕНИЕ 2 . Пусть $S \in S_{\mu}, \pi_{S}$ - соответствующее глобальное сечение $\left.\operatorname{Det}^{*}\right|_{\mathrm{Gr}^{\mu}(V)}$. Через $\left(\begin{array}{cc}\alpha^{a_{1}} & 0 \\ 0 & \nu^{a_{2}}\end{array}\right)^{*}$ обозначим действие $\left(\begin{array}{cc}\alpha^{a_{1}} & 0 \\ 0 & \nu^{a_{2}}\end{array}\right)$ на $H^{0}\left(\operatorname{Gr}^{\mu}(V), \operatorname{Det}^{*}\right)$. Тогда

$$
\left(\begin{array}{cc}
\alpha^{a_{1}} & 0 \\
0 & \nu^{a_{2}}
\end{array}\right)^{*}\left(\pi_{S}\right)=\alpha^{a_{1} \cdot n_{1}(s)} \nu^{a_{2} \cdot n_{2}(s)} \pi_{S} .
$$

ДокАЗАТЕльСТво следует из явного описания действий $\left(\begin{array}{ll}\alpha & 0 \\ 0 & 1\end{array}\right)$ и $\left(\begin{array}{ll}1 & 0 \\ 0 & \nu\end{array}\right)$ на детерминантном расслоении. Это описание получается при помощи прямых вычислений из формул (8)-(10).

Для любого $S \in S_{\mu}$ обозначим $n(S) \stackrel{\text { def }}{=} n_{1}(S)-n_{2}(S)$.

Лемма 4. Пусть $W \in \mathrm{Gr}^{\mu}(V), S(W)=\left\{S \in S_{\mu}: \pi_{S}(W) \neq 0\right\}$. Тогда имеем

1) ecлu $\min _{S \in S(W)} n(S)>-\infty$, mo $l_{1} \cap W \neq 0$;

2) ecлu $\operatorname{rank} A_{W}=1, l_{1} \cap W \neq 0$, mo $\min _{S \in S(W)} n(S)>-\infty$.

ДокАЗАТЕЛЬСтво. Напомним, что

$$
\pi_{S}(W) \neq 0, \text { если и только если } W \oplus A_{S}=V .
$$

Рассмотрим второе утверждение леммы. Если $l_{1} \cap W \neq 0$, то из $\operatorname{dim}_{k} W \cap V_{0}<\infty$ мы имеем $\operatorname{dim}_{k} W \cap l_{1} \cap V_{0}<\infty$. Из rank $A_{W}=1$ получаем

$$
\operatorname{dim}_{k} k((z)) /\left(A_{W}+k[[z]]\right)<\infty
$$

поэтому

$$
\operatorname{dim}_{k} l_{1} /\left(W \cap l_{1}+V_{0} \cap l_{1}\right)<\infty .
$$

Теперь пусть некоторое $S^{\prime} \in S(W)$, тогда $W \oplus A_{S^{\prime}}=V$. Отсюда $W \cap A_{S^{\prime}}=0$. Поэтому

$$
\left(W \cap l_{1}\right) \cap\left(A_{S^{\prime}} \cap l\right)=0 .
$$

Из (13) мы получаем

$$
n_{1}\left(S^{\prime}\right) \geqslant \operatorname{dim}_{k} W \cap l_{1} \cap V_{0}-\operatorname{dim}_{k} l_{1} /\left(W \cap l_{1}+V_{0}+l_{1}\right) .
$$

Из (11) имеем $n\left(S^{\prime}\right)=2 n_{1}\left(S^{\prime}\right)-\mu$. Поэтому

$$
n\left(S^{\prime}\right) \geqslant 2\left(\operatorname{dim}_{k} W \cap l_{1} \cap V_{0}-\operatorname{dim}_{k} l_{1} /\left(W \cap l_{1}+V_{0} \cap l_{1}\right)\right)-\mu .
$$

Второе утверждение леммы доказано.

Рассмотрим теперь первое утверждение леммы. Из $\min _{S \in S(W)} n(S)>-\infty$ мы видим, что $\min _{S \in S(W)} n_{1}(S)>-\infty$. Пусть

$$
\tau=\min \left\{0, \min _{S \in S(W)} n_{1}(S)\right\}-1 .
$$


Для любого $m>0$ определим

$$
B_{m}=z^{\tau} k[[z]] \oplus z^{m+\mu} k[[z]] \subset V .
$$

Зафиксируем некоторое целое число $m_{0}>\max \{0,-\mu,-\tau\}$ так, что

$$
W \cap\left(z^{m_{0}+\mu} V_{0}\right)=0, \quad V /\left(W+z^{-m_{0}} V_{0}\right)=0 .
$$

Мы покажем, что для каждого целого $m>m_{0}$

$$
W \cap B_{m} \neq 0 .
$$

Для этой цели рассмотрим образы $\widetilde{W}$ подпространства $W \cap\left(z^{-m} V_{0}\right)$ и $\widetilde{l_{1}}$ подпространства $l_{1} \cap\left(z^{-m} V_{0}\right)$ в конечномерном грассмановом многообразии

$$
\operatorname{Gr}\left(2 m+\mu, z^{-m} V_{0} / z^{m+\mu} V_{0}\right)
$$

Теперь (15) эквивалентно следуюшему:

$$
\left(\widetilde{W} \cap \widetilde{l_{1}}\right) \cap\left(\left(z^{\tau} V_{0} / z^{m+\mu} V_{0}\right) \cap \tilde{l_{1}}\right) \neq 0
$$

Последняя формула следует из сравнения размерностей конечномерных векторных пространств:

$$
\begin{gathered}
\operatorname{dim}_{k} \tilde{l_{1}}=2 m+\mu, \\
\operatorname{dim}_{k}\left(z^{\tau} V_{0} / z^{m+\mu} V_{0}\right) \cap \tilde{l_{1}}=m+\mu-\tau, \\
\operatorname{dim}_{k} \widetilde{W} \cap \widetilde{l_{1}}>m+\tau,
\end{gathered}
$$

где (16) верна по следующей причине.

Если

$$
\operatorname{dim}_{k} \widetilde{W} \cap \widetilde{l_{1}} \leqslant m+\tau
$$

то мы найдем $k$-векторное подпространство $K \subset \tilde{l_{1}}$ такое, что $K$ есть линейная оболочка элементов $\left\{z^{i_{k}}\right\}$ для некоторых целых $i_{k}$ и $\widetilde{l_{1}}=\left(\widetilde{W} \cap \widetilde{l_{1}}\right) \oplus K$. Тогда мы можем найти $\widetilde{L} \in \operatorname{Gr}\left(2 m+\mu, z^{-m} V_{0} / z^{m+\mu} V_{0}\right)$ такое, что $\widetilde{L}$ является линейной оболочкой элементов $\left\{z^{i_{l}}\right\}$ для некоторых целых чисел $i_{l}, \widetilde{L} \cap \widetilde{l_{1}}=K$ и

$$
z^{-m} V_{0} / z^{m+\mu} V_{0}=\widetilde{W} \oplus \widetilde{L}
$$

Пусть $L-$ прообраз пространства $\widetilde{L}$ в $z^{-m} V_{0}$. Тогда сушествует плюккерова координата $S^{\prime} \in S_{\mu}$ такая, что $L=A_{S^{\prime}}$. Тогда по конструкции

$$
V=W \oplus A_{S^{\prime}}
$$

и из (17) $n_{1}\left(S^{\prime}\right) \leqslant \tau<\min _{S \in S(W)} n_{1}(S)$. Последняя формула противоречит (18). Поэтому формула (16) доказана. Отсюда получаем, что формула (15) верна.

Теперь из условия фредгольмовости на $W$ мы имеем $\operatorname{dim}_{k} W \cap\left(z^{\tau} V_{0}\right)<\infty$. Из (15) имеем непустую фильтрацию $W \cap B_{m}$ для всех достаточно больших целых $m$. Последнее эквивалентно следующему:

$$
\left(W \cap\left(z^{\tau} V_{0}\right)\right) \cap l_{1} \neq 0 .
$$

В самом деле, предположим противное. Тогда выберем конечный $k$-базис $e_{1}, \ldots, e_{n}$ в $W \cap z^{\tau} V_{0}$ такой, что $\nu_{2}\left(e_{i}\right) \neq \nu_{2}\left(e_{j}\right)$ для всех пар $i, j$. (Здесь $\nu_{2}\left(e_{k}\right)=p$, если $e_{k} \in B_{p}$, но $e_{k} \neq B_{p+1}$.) Тогда для всех $m>\max _{i} \nu_{2}\left(e_{i}\right)$ мы имеем $W \cap B_{m}=0$. Это противоречие завершает доказательство леммы 4. 
Лемма 5. Пусть $W \in \mathrm{Gr}^{\mu}(V), \operatorname{rank} A_{W}=1$ и выполняется 1) или 2) предыдущей леммы, тогда

$$
\min _{S \in S(W)} n(S)=2\left(\operatorname{dim}_{k} W \cap l_{1} \cap V_{0}-\operatorname{dim}_{k} l_{1} /\left(W \cap l_{1}+V_{0} \cap l_{1}\right)\right)-\mu .
$$

ДокАЗАТЕЛЬство. Из доказательства (14) мы получаем, что левая сторона (19) больше или равна правой стороне (19).

Зафиксируем теперь некоторое целое $m>0$ так, что

$$
W \cap\left(z^{m+\mu} V_{0}\right)=0, \quad V /\left(W+z^{-m} V_{0}\right)=0 .
$$

Рассмотрим образы $\widetilde{W}$ пространства $W \cap\left(z^{-m} V_{0}\right)$ и $\widetilde{l_{1}}$ пространства $l_{1} \cap\left(z^{-m} V_{0}\right)$ в $z^{-m} V_{0} / z^{m+\mu} V_{0}$.

Тогда найдем $k$-векторное подпространство $K \subset \widetilde{l_{1}}$ такое, что $K$ есть линейная оболочка элементов $\left\{z^{i_{k}}\right\}$ для некоторых целых $i_{k}$ и

$$
\widetilde{l_{1}}=\left(\widetilde{W} \cap \widetilde{l_{1}}\right) \oplus K
$$

Тогда мы можем найти $\widetilde{L} \subset z^{-m} V_{0} / z^{m+\mu} V_{0}$ такое, что $\widetilde{L}$ является линейной оболочкой элементов $\left\{z^{i_{l}}\right\}$ для некоторых целых $i_{l}, \widetilde{L} \cap \widetilde{l_{1}}=K$ и

$$
z^{-m} V_{0} / z^{m+\mu} V_{0}=\widetilde{W} \oplus \widetilde{L}
$$

Через $L$ обозначим прообраз пространства $\widetilde{L}$ в $z^{-m} V_{0}$. Тогда сушествует некотоpoe $S^{\prime} \in S_{\mu}$ такое, что $L=A_{S^{\prime}}$ и $V=W \oplus A_{S^{\prime}}$. Из (20) и (21) мы получаем $n\left(S^{\prime}\right)=n(W)$. Лемма 5 доказана.

\section{§3. Стабильные и нестабильные точки}

Для пучков без кручения на кривой $C$ можно определить понятие “стабильности” и “полустабильности” (см., например, [6].) Напомним эти определения.

ОПРЕДЕЛЕНИЕ 1. Пучок без кручения $\mathscr{F}$ ранга 2 на кривой $C$ называется $n o-$ лустабильнылм пучком, если для любого подпучка $\mathscr{G} \in R(\mathscr{F})$

$$
2 \chi(\mathscr{G}) \leqslant \chi(\mathscr{F})
$$

ОПРЕДЕЛЕНИЕ 2. Пучок без кручения $\mathscr{F}$ ранга 2 на кривой $C$ называется $c m a-$ бильным пучком, если для любого подпучка $\mathscr{G} \in R(\mathscr{F})$

$$
2 \chi(\mathscr{G})<\chi(\mathscr{F})
$$

ТеОрема 1. Пусть $W=K\left(C, p, \mathscr{F}, t_{p}, e_{p}\right)$. Тогда пучок $\mathscr{F}-$ не $($ полу $)$ стабильный пучок, если и только если существует некоторый элемент $g \in$ $\mathrm{SL}(2, k[[z]])$ такой, что для любого $S \in S(g W) n(S) \geqslant 0$ (>0). 
ДоКАЗАТЕЛЬСТво следует из предложения 1 , лемм $2,4,5$ и следующих очевидных утверждений:

1) группа $\mathrm{SL}(2, k[[z]])$ действует транзитивно на множестве всех 1-мерных $k((z))$-векторных подпространств в $V$;

2) группа $\mathrm{SL}(2, k[[z]])$ переводит подпространство $V_{0}$ в себя.

ЗАмЕчАНИЕ. Из леммы 5 и формулы (5) мы можем также получить численные данные нестабильности пучка.

ПримеР 1 (см. также [1; prop. 3.8]). Пусть $\mu=2 \nu$ для некоторого целого $\nu$ и $W=K\left(C, p, \mathscr{F}, t_{p}, e_{p}\right)$ принадлежит большой клетке в $\mathrm{Gr}_{V}^{\mu}$, т.е.

$$
\pi_{0(\mu)}(W) \neq 0,
$$

тогда пучок $\mathscr{F}$ - полустабильный пучок. В самом деле, если условие $(22)$ выполняется, то $W \oplus z^{\nu} V_{0}=V$ и для любого $g \in \mathrm{SL}(2, k[[z]]), g\left(z^{\nu} V_{0}\right)=z^{\nu} V_{0}$, $g W \oplus z^{\nu} V_{0}=V$. Поэтому $\pi_{0(\mu)}(g W) \neq 0$. Но $n(0(\mu))=0$ и мы применяем теореMy 1.

Мы можем обобшить пример 1 следуюшим образом.

ПримеР 2. С каждым $W \in \mathrm{Gr}^{\mu}(V)$ можно связать:

1) функцию $T_{W}: \mathbb{Z} \rightarrow\{0,1,2\}$ такую, что $T_{W}(i)=2$ для $i \ll 0, T_{W}(i)=0$ для $i \gg 0$ и для остальных $i T_{g W}(i)=T_{W}(i)$ для любых $g \in \mathrm{SL}(2, k[[z]])$, где

$$
T_{W}(i) \stackrel{\text { def }}{=} \operatorname{dim}_{k}\left(W \cap z^{i} V_{0}\right) /\left(W \cap z^{i+1} V_{0}\right) ;
$$

2) точку

$$
x_{W}=\left(x_{W, i_{1}}, \ldots, x_{W, i_{l}}\right) \in\left(\mathbb{P}_{k}^{1}\right)^{l_{W}},
$$

где $i_{1}, \ldots, i_{l_{W}}$ все целые числа такие, что $T_{W}\left(i_{j}\right)=1$ и точка $x_{W, i_{j}} \in \mathbb{P}_{k}^{1}$ определяется как 1-мерное $k$-векторное пространство $\left(W \cap z^{i_{j}} V_{0}\right) /(W \cap$ $\left.z^{i_{j}+1} V_{0}\right)$ в 2-мерном $k$-векторном пространстве $z^{i_{j}} V_{0} / z^{i_{j}+1} V_{0}$.

Заметим, что все $\mathbb{P}_{k}^{1}$ из (23) имеют канонические однородные координаты, индуцированные $z^{i_{j}} \oplus 0 \in V$ и $0 \oplus z^{i_{j}} \in V$ для соответствующих $j$. При помощи этих координат мы можем отождествить все $\mathbb{P}_{k}^{1}$ из $(23)$ и рассмотреть $x_{W}$ как набор $l_{W}$ упорядоченных точек на прямой.

Сушествует естественное диагональное действие группы $\mathrm{SL}(2, k)$ в $\left(\mathbb{P}_{k}^{1}\right)^{l_{W}}$. Рассмотрим вложение Сегре $\left(\mathbb{P}_{k}^{1}\right)^{l_{W}} \hookrightarrow \mathbb{P}_{k}^{2 l_{W}-1}$. Существует единственное линейное действие $\mathrm{SL}(2, k)$ в $\mathbb{P}_{k}^{2 l_{W}-1}$, которое преврашает вложение Сегре в $\mathrm{SL}(2, k)$-морфизм $\left(l_{W}\right.$-кратное тензорное представление $\left.\mathrm{SL}(2, k)\right)$. Kроме того, группа $\mathrm{SL}(2, k)$ - редуктивная группа. Поэтому геометрическая теория инвариантов определяет (полу)стабильные точки на $\left(\mathbb{P}_{k}^{1}\right)^{l_{W}}$ по отношению к $\mathrm{SL}(2, k)$-действию и вложению Сегре. Из [6] мы имеем следующее явное описание: точка $x \in\left(\mathbb{P}_{k}^{1}\right)^{l_{W}}-($ полу)стабильная точка, если и только если никакая точка $\mathbb{P}_{k}^{1}$ не встречается как компонента точки $x$ больше или равно (больше) $l_{W} / 2$ раз.

Сформулируем теперь следующее предложение. 
ПРЕДЛОЖЕНИЕ 3. Пусть $W=K\left(C, p, \mathscr{F}, t_{p}, e_{p}\right)$. Тогда

1) если $1 \notin \operatorname{Im} T_{W}$, то пучок $\mathscr{F}$ - полустабильный пучок;

2) если точка $x_{W} \in\left(\mathbb{P}_{k}^{1}\right)^{l_{W}}-($ полу) стабильная точка по отношению $к$ $\mathrm{SL}(2, k)$-действию и влохсению Сегре, то пучок $\mathscr{F}-($ полу) стабильный пучок.

ДокАЗАТЕльство. В самом деле, если $1 \notin \operatorname{Im} T_{W}$, то определим $k$-векторное подпространство $M \subset V$ как линейную оболочку элементов $\left\{\left\{z^{i} \oplus 0\right\},\left\{0 \oplus z^{i}\right\}\right.$ : $\left.T_{W}(i)=2\right\}$. Мы можем найти $S^{\prime} \in S_{\mu}$ такое, что $M=H_{S^{\prime}}$. Из конструкции $W \oplus A_{S^{\prime}}=V$. Отсюда $\pi_{S^{\prime}}(W) \neq 0$. Для любого $g \in \mathrm{SL}(2, k[[z]])$ имеем $T_{g W}=T_{W}$. Поэтому $\pi_{S^{\prime}}(g W) \neq 0$. Из теоремы 1 и $n\left(S^{\prime}\right)=0$ мы видим, что пучок $\mathscr{F}-$ полустабильный пучок.

Рассмотрим теперь случай 2). Пусть $x_{W}=\left(x_{W, i_{1}}, \ldots, x_{W, i_{l}}\right) \in\left(\mathbb{P}_{k}^{1}\right)^{l_{W}}$. Для каждого $k \in\left\{i_{1}, \ldots, i_{l_{W}}\right\}$ мы можем выбрать элемент $z_{k} \in V$, совпадающий с $\left\{z^{k} \oplus 0\right\}$ или $\left\{0 \oplus z^{k}\right\}$ так, что образ $k$-прямой $k \cdot z_{k}$ в $z^{k} V_{0} / z^{k+1} V_{0}$ не совпадает с $x_{W, k}$. Определим $k$-векторное подпространство $M \subset V$ как линейную оболочку элементов $\left\{z_{k}: k \in\left\{i_{1}, \ldots, i_{l_{W}}\right\}\right\}$ и $\left\{\left\{z^{i} \oplus 0\right\},\left\{0 \oplus z^{i}\right\}: T_{W}(i)=2\right\}$. Тогда существует $S^{\prime} \in S_{\mu}$ такое, что $M=H_{S^{\prime}}$ и $W \oplus A_{S^{\prime}}=V$, т.е. $\pi_{S^{\prime}}(W) \neq 0$. Более того, если точка $x_{W} \in\left(\mathbb{P}_{k}^{1}\right)^{l_{W}}-$ (полу)стабильная точка, то из явного описания таких точек мы можем выбрать $z_{k} \in V$ так, что

$$
n\left(S^{\prime}\right)<0 \quad(\leqslant 0)
$$

Действие групшы $\mathrm{SL}(2, k)$ на $\left(\mathbb{P}_{k}^{1}\right)^{l_{W}}$ индуцировано действием групшы $\mathrm{SL}(2, k[[z]])$ на $V$. Поэтому точка $x_{g W} \in\left(\mathbb{P}_{k}^{1}\right)^{l_{g} W}-$ (полу)стабильная точка для любого $g \in \mathrm{SL}(2, k[[z]])$. Теперь из этого факта, формулы (24) и теоремы 1 мы получаем (полу)стабильность пучка $\mathscr{F}$.

ЗАмЕчАнИЕ. Предположим, что точка $x \in\left(\mathbb{P}_{k}^{1}\right)^{l}$ не является полустабильной точкой по отношению к $\mathrm{SL}(2, k)$-действию и вложению Сегре. Тогда легко построить квинтеты $\left(C, p, \mathscr{F}, t_{p}, e_{p}\right)$ со стабильными, полустабильньми и нестабильными пучками $\mathscr{F}$ так, что для $W=K\left(C, p, \mathscr{F}, t_{p}, e_{p}\right)$ мы имеем $l_{W}=l_{\text {и }} x_{W}=x$.

ЗАмечАниЕ. Можно определить клеточное разбиение $\mathrm{Gr}^{\mu}(V)$, индексированное множеством $S_{\mu}$ (см. [5]): $W \in \operatorname{Gr}^{\mu}(V)$ лежит в клетке $S=\left\{s_{-\mu+1}, s_{-\mu+2}, \ldots\right\}$ при выполнении следующих условий:

1) если $\pi_{S}(W) \neq 0$;

2) для любого $S^{\prime}=\left\{s_{-\mu+1}^{\prime}, s_{-\mu+2}^{\prime}, \ldots\right\}$, если $\pi_{S^{\prime}}(W) \neq 0$, то $\left\{s_{-\mu+1}^{\prime} \geqslant\right.$ $\left.s_{-\mu+1}, s_{-\mu+2}^{\prime} \geqslant s_{-\mu+2}, \ldots\right\}$.

(Из этих условий такое $S \in S_{\mu}$ определяется единственным образом для каждого $W \in \mathrm{Gr}^{\mu}(V)$.) Нетрудно видеть, что случай 1$)$ предложения 3 соответствует следуюшему утверждению: если $W=K\left(C, p, \mathscr{F}, t_{p}, e_{p}\right)$ принадлежит клетке $S \in S_{\mu}$ такой, что $H_{S} \cap l_{1}=H_{S} \cap l_{2}$, то пучок $\mathscr{F}$ полустабилен.

Заметим теперь, что действие групшы $\mathrm{SL}(2, k[[z]])$ на $\mathrm{Gr}^{\mu}(V)$ оставляет $W=$ $K\left(C, p, \mathscr{F}, t_{p}, e_{p}\right) \in \mathrm{Gr}^{\mu}(V)$ в образе отображения Кричевера и не меняет кривой $C$, точки $p$, локального параметра $t_{p}$ и пучка $\mathscr{F}$. Итак, это действие есть действие только на "базисах $e_{p}$ ". Поэтому, если бы мы хотели получить что-нибудь как 
“пространство модулей” пучков на $C$, нам было бы нужно как-то отфакторизовать по действию групшы $\mathrm{SL}(2, k[[z]])$ на $\mathrm{Gr}^{\mu}(V)$.

$\mathrm{C}$ другой стороны, по лемме 3 группа $\mathrm{SL}(2, k[[z]])$ действует на Det $\left.\right|_{\mathrm{Gr}^{\mu}(V)}$. Более того, нетрудно понять, что это действие задает нам бесконечномерное представление группы $\mathrm{SL}(2, k[[z]])$ в $k$-векторном пространстве $\Pi\left(S_{\mu}\right)$. (Более точно, это представление есть ограничение представления групшы $\widehat{\mathrm{GL}_{0}}\left(V, V_{0}\right)$ в пространстве $\Pi\left(S_{\mu}\right)$, которое может быть получено тем же способом и является бесконечномерным wedge-представлением (подробнее см. [7] и [8]).) Отсюда мы получаем линейное действие группы $\mathrm{SL}(2, k[[z]])$ на $\mathbb{P}\left(\Pi\left(S_{\mu}\right)^{*}\right)$. Более того, нетрудно понять, что на $\mathrm{SL}(2, k[[z]])$ можно ввести структуру афинной групповой схемы, представляющей функтор $S \longmapsto \mathrm{SL}(2, k[S][[z]])(S-$ произвольная $k$-алгебра). Тогда описанное выше действие на $\mathbb{P}\left(\Pi\left(S_{\mu}\right)^{*}\right)=\operatorname{Proj}\left(\operatorname{Sym}\left(\Pi\left(S_{\mu}\right)\right)\right)$ является корректно определенным действием в категории схем. Аналогично, действие $\mathrm{SL}(2, k[[z]])$ на $\mathrm{Gr}^{\mu}(V)$ является корректно определенным действием в категории схем. И по конструкции эти действия совпадают при плюккеровом вложении. Эти аргументы приводят нас к следующим определениям.

Для каждого $W \in \mathrm{Gr}^{\mu}(V)$ через $\widehat{W}$ обозначим некоторьй ненулевой элемент из $\Pi\left(S_{\mu}\right)^{*}$ такой, что образ $\widehat{W}$ в $\mathbb{P}\left(\Pi\left(S_{\mu}\right)^{*}\right)$ совпадает с образом $W$ при плюккеровом вложении.

Пусть

$$
T=\left\{\left(\begin{array}{cc}
\lambda & 0 \\
0 & \lambda^{-1}
\end{array}\right) \in \mathrm{SL}(2, k[[z]]), \lambda \in k^{*}\right\}
$$

- 1-параметрическая подгруппа: $k^{*} \rightarrow \mathrm{SL}(2, k[[z]])$. Для любого $g \in \mathrm{SL}(2, k[[z]])$ определим группу ${ }^{g} T=g T g^{-1}$. Пусть ${ }^{g} T(\lambda)=g\left(\begin{array}{cc}\lambda & 0 \\ 0 & \lambda^{-1}\end{array}\right) g^{-1}$ - элемент из ${ }^{g} T$.

Далее для удобства отождествим очевидным способом $k^{*}$ с подмножеством в $\mathbb{P}_{k}^{1}$. $\mathrm{B}$ частности, для любого $\lambda \in k$ мы отождествляем $\lambda$ с точкой $(1, \lambda)$ в $\mathbb{P}_{k}^{1}$ и пишем $\infty$ для внешней точки $(0,1)$. Отметим, что морфизм $k^{*} \rightarrow \Pi\left(S_{\mu}\right)^{*}: \lambda \mapsto{ }^{g} T(\lambda) \widehat{W}$ может как расширяться, так и не расширяться на точки $0, \infty$, но если расширение существует (для одной или обоих точек), то оно единственно. (Это следует из валюативного критерия отделимости.) Таким образом, мы можем определить очевидньм способом выражения $\lim _{\lambda \rightarrow 0}{ }^{g} T(\lambda) \widehat{W}$ и $\lim _{\lambda \rightarrow \infty}{ }^{g} T(\lambda) \widehat{W}$.

ОПРЕДЕЛЕНИЕ 3. Замкнутая точка $W \in \mathrm{Gr}^{\mu}(V)$ - полустабильная точка по отношению $\kappa$ 1-параметрической подгруппе ${ }^{g} T$, если для $x=0$ и $x=\infty$ $\lim _{\lambda \rightarrow x}{ }^{g} T(\lambda) \widehat{W}$ не сушествует или $\lim _{\lambda \rightarrow x}{ }^{g} T(\lambda) \widehat{W} \neq 0$.

ОПРЕДЕЛЕНИЕ 4. Замкнутая точка $W \in \mathrm{Gr}^{\mu}(V)$ - стабильная точка по отношению $\kappa$ 1-параметрической подгруппе ${ }^{g} T$, если для $x=0$ и $x=\infty$ $\lim _{\lambda \rightarrow x}{ }^{g} T(\lambda) \widehat{W}$ не сушествует.

Используя теперь эти определения и предложение 2, мы можем следующим способом переформулировать теорему 1. (Сравните с конечномерной ситуацией в [6].)

Tеорема 2. Рассмотрим любой квинтет $\left(C, p, \mathscr{F}, t_{p}, e_{p}\right), \chi(\mathscr{F})=\mu$. Тогда пучок $\mathscr{F}$ - (полу)стабильный пучок на кривой $C$, если и только если $K\left(C, p, \mathscr{F}, t_{p}, e_{p}\right) \in \mathrm{Gr}^{\mu}(V)-($ полу $)$ стабильная точка по отношению $\kappa$ 1-параметрическим подгруппам ${ }^{g} T$ для всех $g \in \mathrm{SL}(2, k[[z]])$. 


\section{Список литературы}

1. Mulase $M$. Category of vector bundles on algebraic curves and infinite dimensional grassmanians // Internat. J. Math. 1990. V. 1. № 3. P. 293-342.

2. Previato E., Wilson G. Vector bundles over curves and solutions of the KP equations // Proc. Sympos. Pure Math. 1989. V. 49. P. 553-569.

3. Осипов Д. В. Отображение Кричевера и (полу)стабильные пучки на кривых // УМН. 1999. Т. 54. № 3. С. 177-178.

4. Álvarez Vázquez A., Munoz Porras J. M., Plaza Martín F. J. The algebraic formalism of soliton equations over arbitrary base fields // Workshop on abelian varieties and theta functions. Morelia, México, July 8-27, 1996. Proceedings / ed. R. Rodriguez et al. México: Soc. Mat. Mexicana, 1998. P. 3-40. (Aportaciones Mat. Invest. V. 13.)

5. Прессли Э., Сигал Г. Группыпетель. М.: Мир, 1990.

6. Newstead P.E. Lectures on introduction to moduli problems and orbit spaces. Berlin: Springer-Verlag, 1978. (Tata Inst. Fund. Res. Lect. Math. and Phys. Math. V. 51.)

7. Kac V.G., Peterson D. H. Lectures on the infinite wedge-representation and the MKP hierarchy // Systèms dynaniques non linéares: intégrabilité et comportement qualitatif, Sémin. Math. Supér., Sémin. Sci. OTAN (NATO Adv. Study Inst.). V. 102, 1986. P. 141-183.

8. Kac V.G., Raina A.K. Bombay lectures on highest weight representations. Singapore: World Sci., 1987.

Математический институт им. В. А. Стеклова РАН,

Поступила в редакцию

Университет Гумбольдта, г. Берлин

10.04 .2003

E-mail: d_osipov@mi.ras.ru 